\section{EL TRATAMIENTO NUMÉRICO DE LA REALIDAD. REFLEXIONES SOBRE LA IMPORTANCIA ACTUAL DE LA ESTADÍSTICA EN LA SOCIEDAD DE LA INFORMACIÓN}

\author{
Toni Monleón-Getino \\ Departamento de Estadística. Universidad de Barcelona. \\ Avda. Diagonal 645. 08028 Barcelona. \\ amonleong@ub.edu.Tf: 93-402.15.60 (678-329864)
}

\author{
THE NUMERICAL TREATMENT OF \\ THE REALITY. THOUGHTS ABOUT \\ THE NOWADAYS IMPORTANCE \\ OF THE STATISTICS IN THE \\ INFORMATION SOCIETY
}

\begin{abstract}
The statistic has become an effective method to accurately describe the data values in economic, political, social, psychological, medical, biological and physical as a tool to correlate and analyze data. The statistical work is not only to obtain, collect or tabulate the data, but on the whole process of interpreting that information alone or with other specialists. Nowadays is impossible to understand the Information Society without statistical concern is the current status of mathematics and specifically of statistics and their subsequent translation into society because of its indispensability despite a lack of vocation among young people study and use, either by its mathematical difficulty, ignorance or other reasons. This article aims to reflect on the importance of it in today's society that makes it good numerical reality.
\end{abstract}

KEY WORDS: Statistic; information society; mathematics; uncertanty; science.

\section{¿QUÉ IMPORTANCIA TIENE LA ESTADISTICA} EN LA SOCIEDAD DE LA INFORMACIÓN?

La ciencia intenta conocer los fenómenos naturales y mejorarlos. Tal entendimiento puede explicarse mediante un juicio de abstracción y frecuentemente se expresa en términos de leyes, axiomas y teorías que permiten predecir eventos futuros sin precisar ciertos límites de exactitud (Rubio de Juan, 2001).

En la sociedad de la información las actividades humanas están basadas en predicciones, en ella la creación, distribución y tratamiento de la información forman parte de todas las actividades sociales y económicas que forman parte
RESUMEN: La estadistica se ha convertido en un método práctico para describir los valores de datos económicos, políticos, sociales, psicológicos, médicos, biológicos y físicos, como herramienta para relacionar y analizar dichos datos. El trabajo del estadístico no consiste sólo en obtener, reunir o tabular los datos, sino sobre todo el proceso de interpretación de esa información solo o en colaboración con los expertos en cada ámbito. A pesar de que es imposible entender la Sociedad de la Información sin la estadística, es preocupante la situación actual de las ciencias matemáticas y más concretamente de la estadística, ya que a pesar de su imprescindibilidad existe una falta de vocación entre los jóvenes por su estudio y uso, ya sea por su dificultad matemática, desconocimiento u otros motivos. Este artículo pretende reflexionar sobre la importancia de la misma en la sociedad actual que permite tratar numéricamente la realidad.

PALABRAS CLAVE: Estadística; sociedad de la información; matemáticas; incertidumbre; ciencia.

de nuestra vida y que constituyen nuestro medio ambiente. La abundancia de información nos rodea, en ocasiones sobrepasa nuestra capacidad, procede de fuentes diversas y es necesaria para tomar decisiones en un ambiente de incertidumbre (ANECA, 2004). Cualquier toma de decisiones lleva implícita la incertidumbre: ¿qué carrera estudiar? ¿qué producto comprar? ¿dónde invertir? Cuidarse de tomar decisiones puede considerarse como la alternativa para evitar errores, sin embargo, no podríamos hablar de progreso si aceptásemos esa forma de proceder. Por ello, la mejor forma de proceder a la hora de tomar decisiones es optimizar, recortar el riesgo que cualquier alternativa lleva implícita. Una conocida chanza estadística afirma que "La probabilidad de tener un accidente de tráfico aumenta con el tiempo que te pases en la calle. Por tanto, cuanto más 
rápido circules, menor es la probabilidad de que tengas un accidente" Quizás en este caso deberíamos parar a reflexionar más sobre las consecuencias de la decisión.

La Estadistica es una herramienta ineludible para tratar de cuantificar la incertidumbre y su uso es inevitable en la exploración del conocimiento. Los conceptos de incertidumbre y aleatoriedad han ofuscado a la humanidad desde el origen de los tiempos, así las sociedades primitivas, aunque de manera rudimentaria, ya contaban y procesaban información numérica, como el número de cabezas de ganado que poseian. Actualmente y muy frecuentemente, nos acecha la incertidumbre en el entorno físico y social en el que vivimos, más tanto en cuanto cuánta más información y más incertidumbre sufrimos en la esta era de la información y de la informática. Soportamos las incertidumbres de la naturaleza y sufrimos sus catástrofes (Rubio de Juan, 2001), sólo hay que recordar la impredictibilidad de los terremotos.

Desde el punto de vista filosófico, algunos teólogos contemporáneos discuten que nada es aleatorio excepto Dios, ya que es la causa de todo lo que sucede, pero en la antigüedad los filósofos coetáneos de Aristóteles no contemplaban la posibilidad de estudiar el azar o medir su incertidumbre, así "Las leyes científicas no avanzan mediante un principio dictatorial o pueden justificarse mediante fe o filosofía medieval; La Estadística es el único tribunal hacia el nuevo conocimiento." (Mahalanobis, 1954). Rao (1989) destaca la necesidad de buscar estrategias en el análisis de datos para extraer información relevante de lo observado y ocuparse de la incertidumbre.

Algunos discuten que la aleatoriedad no debe confundirse con la impredictibilidad práctica, así algunos sistemas matemáticos, pueden verse como aleatorios; sin embargo son de hecho impredecibles, debiéndose a una dependencia sensible de las condiciones iniciales: mientras el incremento de la población humana es bastante predecible, ya que existen estadísticas anuales, locales, etc., el intervalo real de los nacimientos y muertes individuales no puede predecirse, siendo muy común ese tipo de aleatoriedad en el entorno que nos rodea, aunque a a pequeña escala.

En ciertas tecnologías como la criptografía, donde se aplican números aleatorios, requieren de la impredictibilidad. En otras como la simulación, es esencial la aleatoriedad es- tadística, pero también de la predictibilidad (si se ejecutan repetidamente simulaciones o pruebas de reconocimiento, puede sea útil poder volver a ejecutar el modelo con la entrada aleatoria exacta (semilla aleatoria) cuando sea requerido. (Wikipedia, 2009b).

El pensamiento de la sociedad actual donde la información se genera en tiempo real y de manera industrial, se fundamente en el racionalismo empírico, pensamiento desarrollado esencialmente por René Descartes (1596-1650) y expuesto esencialmente en su obra cumbre, el Discurso del Método (1637), donde destaca la facultad natural que todo hombre tiene para razonar, realizando una aproximación a la ciencia, la medicina, las matemáticas y la geometría: "En los escritos de los poetas hay sentencias más serias que en los de los filósofos. La razón es que los poetas las escribieron movidos por el entusiasmo y el poder de la imaginación. En cada uno de nosotros existen, cual pedernales, chispas de conocimiento ocultas. Los filósofos las manifiestan a través de la razón; los poetas las exteriorizan por medio de la imaginación, y son mucho más brillantes". Así actualmente los procesos de toma de decisiones están sometidos a procesos de análisis estadísticos, previa la cuantificación, valoración o estimación de las causas y los efectos de las decisiones citadas (ANECA, 2004).

Del racionalismo empírico nace la aspiración por saber y los esfuerzos para la cuantificación de los fenómenos, también para su clasificación y sistematización, para describir o para inventar clases y órdenes (Rubio de Juan, 2001) tan común en todos los ámbitos del conocimiento.

La estadística es la ciencia que utiliza como instrumento de trabajo las matemáticas y el cálculo de probabilidades, estudiando y prediciendo el comportamiento de los fenómenos que dependen del azar, con los que detectar comportamientos futuros, pero en muchos casos no queda claro su origen, definición y fundamentación, por lo que a continuación se exponen muy brevemente.

\section{¿Pero qué es la estadistica Y EN QUÉ SE FUNDAMENTE?}

"La probabilidad de tener un accidente de tráfico aumenta con el tiempo que pasas en la calle. Por tanto, cuanto mas 
rápido circules, menor es la probabilidad de que tengas un accidente. El $33 \%$ de los accidentes mortales involucran a alguien que ha bebido. Por tanto, el $67 \%$ restante ha sido causado por alguien que no había bebido. A la vista de esto y de lo anterior, está claro que la forma más segura de conducir es ir borracho y a gran velocidad".

Cabe decir que la inspiración de este apartado ha sido la lectura de la lección inaugural del curso académico 20012002 de la Universidad de Extremadura, titulado "Estadística, certeza e incertidumbre" de la Dra M. ${ }^{\text {a }}$ Asunción Rubio de Juan (Rubio de Juan, 2001), desde mi punto de vista excelente como reflexión filosófica e histórica.

La palabra 'estadistica' procede del latín statisticum collégium ('consejo de Estado') y de su derivado italiano statista ('hombre de Estado' o 'político') y del. El término alemán statistik fue primeramente introducido por Gottfried Achenwall (1719-1772), filósofo y estadístico prusiano que se considera uno de los padres del término y lo introdujo en el estudio titulado "Staatswissenschaft der vornehmen Europäischen Reiche und Republiken", "Estadística" designaba originalmente el análisis de datos del Estado, es decir, "la ciencia del Estado" (también llamada "aritmética política" de su traducción directa del inglés). No fue hasta el siglo XIX cuando el término "estadistica" adquirió el significado de recolectar y clasificar datos, cuando fue introducido en Inglaterra por el inglés Sir John Sinclair (1754-1835) con su trabajo "Statistical Account of Scotland" (1791-1799) (Wikipedia, 2009a). No hay que confundir tampoco al "estadista", hombre de Estado con el "estadístico", hombre de los datos. A principios del siglo XIX, la palabra estadística adopta un significado más generalizado hacia la recolección y clasificación de cualquier tipo de datos cuantitativos.

En su origen, la estadística estuvo asociada a datos que iban a ser utilizados por el gobierno y cuerpos de funcionarios administrativos, frecuentemente centralizados. En particular, los censos proveen información regular acerca de la población (Wikipedia, 2009a).

Desde los comienzos de la civilización han existido maneras sencillas de estadística, pues ya se utilizaban representaciones gráficas y otros símbolos en pieles, rocas, palos de madera y paredes de cuevas para contar el número de personas, animales o ciertas cosas. Hacia el año 3000 a. C. los babilónicos usaban ya pequeñas tablillas de arcilla para recopilar datos en tablas sobre la producción agrícola y de los géneros vendidos o cambiados mediante trueque. Los egipcios analizaban los datos de la población y la renta del país mucho antes de construir las pirámides (siglo XI a. C.) Los libros bíblicos de Números y Crónicas incluyen, en algunas partes, tratados de estadística. El primero contiene dos censos de la población de Israel y el segundo describe el bienestar material de las diversas tribus judias. En China existían registros numéricos similares con anterioridad al año 2000 a. C. Los griegos clásicos realizaban censos cuya información se utilizaba hacia el 594 a. C. para cobrar impuestos (Wikipedia, 2009a).

Para hablar de qué es la estadística, recuerdo una viñeta del dibujante Antonio Fraguas de Pablo "Forges" en un periódico de ámbito estatal donde dos individuos dialogaban acaloradamente y uno decía a otro "Si la estadística no miente" y el otro lo increpaba diciendo "Miente!", el primero respondia "entonces nada", asi la estadística ha sido definida de muchas maneras informalmente que se refieren a la célebre frase del estadista inglés Benjamín Disraeli (1804-1881): "Hay tres tipos de mentiras: mentiras, condenadas mentiras y mentiras estadísticas" que reflejan un principio negativo de la sociedad ante esta ciencia; otra es aquella que la reduce a la ciencia de los promedios o de los números mágicos que indica que si usted introduce su cabeza en la nevera y los pies en un horno caliente, como media su temperatura es la idónea.

Bromas aparte, esta rama de las matemática se define como "la ciencia con base matemática referente a la recolección, análisis e interpretación de datos, que busca explicar condiciones regulares en fenómenos de tipo aleatorio" (Wikipedia, 2009).

Muchos conjuntos de datos se pueden aproximar, con gran exactitud, utilizando determinadas distribuciones probabilísticas; los resultados de éstas se pueden utilizar para analizar posteriormente los datos. Así la estadística, presenta las mismas bases que el cálculo y el álgebra, aunque dependiendo de la rama del conocimiento en la que se imparta la docencia la base matemática se obvia o simplifica, sin tener en cuenta el uso interesado que se pueda querer hacer de ella. Tanur et al. (1992) en su obra "La Estadística: Una guía de lo desconocido" han recopilado una serie de artículos y ensayos realizados en diversas áreas, cubriendo varios campos de la vida en los cuáles la estadística ha sido utilizada para la resolución de un determinado caso. 
La estadistica estudia poblaciones y efectúa afirmaciones sobre sus parámetros, por tanto no se interesa en lo que ocurre con un individuo concretos de la población, ya que no se conoce exactamente lo que va a sucederle; por ello la estadistica estudia subconjuntos de la población, la muestra, y luego generaliza sus conclusiones a la población completa, que se denomina inferencia. Si se estudiara toda la población completa no estariamos hablando de estadística, y las conclusiones obtenidas serian ciertas con una probabilidad del $100 \%(p=1)$. La muestra ha de ser representativa de la población, sino se produciria un sesgo y por ello generalmente se escogen los individuos al azar dentro de la población, a este proceso se lo denomina muestreo. Así el azar garantiza que la muestra es representativa de la población, pero los resultados obtenidos se enuncian como una probabilidad y por ello las afirmaciones de la estadistica se apunta que son probabilisticas.

Como ciencia la estadística no explica fenómenos ni establece relaciones causales, sino asociaciones estadísticas. Esto, requiere explicaciones adicionales: por ejemplo existe una asociación en ciertas zonas de la Península, entre el aumento de las cigüeñas y el número de nacimiento de niños, pero esto no significa que las cigüeñas sean las causantes de los nacimientos, sino que son relaciones indirectas.

Según Sánchez de Cos (2007) "no podemos prescindir de la estadistica porque ésta nos ayuda a estudiar poblaciones completas acudiendo a una pequeña muestra, ahorrando así recursos y tiempo; asimismo, nuestro desconocimiento de realidades muy complejas nos permite al menos establecer asociaciones estadísticas, que nos pueden proporcionar pistas sobre posibles interpretaciones causales que posteriormente serán experimentadas".

Con respecto a la estadística sanitaria, especialidad en la que trabaja el autor de este trabajo, cabe decir que se utilizó en España por primera vez para solucionar y mejorar la salud pública de los ciudadanos hace más de 100 años. La estadística sanitaria se convirtió en un ideal para la medicina del siglo XIX, y en uno de los fundamentos de la salud pública actual. Sin embargo, en el caso español, las dificultades que encontró el perfeccionamiento para disponer de adecuada organización de la moderna estadística determinaron el avance de las estadísticas demográficosanitarias y la aplicación del método numérico a los regis- tros poblacionales (Bernabe-Mestre, 2007), piénsese que no existían ordenadores y todos los cálculos se realizaban a mano o utilizando tablas aproximadas.

En medicina, como en cualquier otra ciencia (natural, técnica o humanística) se requiere de la investigación, tanto observacional como experimental, y en cada caso es necesario someter los resultados a análisis estadístico para confirmar o rechazar las hipótesis planteadas inicialmente (Salinas, 2007). Las respuestas esperadas por el propio investigador a dicha pregunta son lo que se conoce por hipótesis de estudio. Una vez definida la hipótesis, el investigador debe evaluar su cumplimiento mediante un estudio diseñado con este fin empleando la sistemática del método científico y la metodología estadistica, lo que le permitirá aceptar, modificar o rechazar la hipótesis definida. En función de la aceptación o no de la hipótesis, el investigador formulará la ley en base a un razonamiento de tipo inductivo, es decir generalizará los resultados del estudio a la población de referencia correspondiente.

Las características y contenidos de los estudios o ensayos que se realicen como parte de la investigación de un fenómeno deben estar definidos en un protocolo que establece la razón de ser de un estudio (objetivos, diseño, metodología, análisis previsto de los resultados y condiciones de realización).

La medida de respuesta o respuestas al tratamiento elegidas en el estudio determinarán los métodos estadísticos que se utilizarán durante el análisis de los datos del estudio y también el tamaño de muestra.

Mediante la correcta aplicación del análisis estadístico y de una adecuada planificación se puede determinar si la relación encontrada entre la exposición a un tratamiento y un resultado es casual o por el contrario, está sujeto a una relación no aleatoria que podría establecer una relación de causalidad. La estadística ayuda a conocer el papel del azar en las hipótesis de trabajo, pero no previene de otros errores comunes que se cometen durante la investigación clínica, como son los sesgos de confusión y selección de la muestra.

Por ejemplo, en el ámbito médico, deben realizarse estudios clínicos con grupos de pacientes y no con un solo paciente ya que existe una enorme variabilidad en la respuesta entre 
los diferentes pacientes aunque presenten caracteristicas similares (Dos personas, aunque parezcan iguales pueden responder de manera muy diferente al mismo tratamiento). La valoración del efecto del tratamiento deberá realizarse en una muestra de pacientes para extrapolar posteriormente los resultados al resto de la población.

La formulación de las hipótesis corresponde a la redacción numérica de la pregunta u objetivo del estudio, que tras ser evaluado su resultado permitirá decidir aceptar o rechazar las hipótesis formuladas mediante un contraste de hipótesis. Las hipótesis formuladas en todo estudio son dos, la hipótesis nula o $\mathrm{H}_{0}$ y la hipótesis alternativa o $\mathrm{H}_{\mathrm{A}}$, que son mutuamente excluyentes. La $\mathrm{H}_{0}$ es la hipótesis que se considera cierta antes de iniciar el estudio y en ausencia de resultados del mismo, por lo que el objetivo del estudio será decidir, con los datos obtenidos, si se acepta una hipótesis distinta $\left(H_{A}\right)$. Para la evaluación del cumplimiento o no cumplimiento de $\mathrm{H}_{\mathrm{A}}$, se calcula un valor caracteristico de la muestra, denominado estadístico. A partir de dicho estadístico se define la región crítica o rango de valores y en el caso de que el estadístico esté dentro de la región crítica, podremos aceptar $\mathrm{H}_{\mathrm{A}} \mathrm{Y}$ rechazar $\mathrm{H}_{0}$.

TABLA 1. TIPOS DE ERRORES OBTENIDOS EN EL CONTRASTE DE HIPÓTESIS

\begin{tabular}{|l|l|l|}
\hline & \multicolumn{2}{|l|}{ Resultados reales } \\
\hline $\begin{array}{l}\text { Resultado del } \\
\text { contraste }\end{array}$ & $\begin{array}{l}\mathrm{H}_{\mathrm{A}} \text { falsa y } \mathrm{H}_{0} \\
\text { verdadera }\end{array}$ & $\begin{array}{l}\mathrm{H}_{\mathrm{A}} \text { verdadera y } \mathrm{H}_{0} \\
\text { falsa }\end{array}$ \\
\hline Rechazo de $\mathrm{H}_{\mathrm{A}}$ & Sin error & Error de tipo II $(\beta)$ \\
\hline Aceptación de $\mathrm{H}_{\mathrm{A}}$ & Error de tipo I $(\alpha)$ & Sin error \\
\hline
\end{tabular}

La aceptación o no aceptación de $\mathrm{H}_{\mathrm{A}}$ se basa en criterios estadísticos, por lo que está sujeta a dos tipos de errores distintos, tal y como se describe en la tabla 1. La aceptación de $H_{A}$ cuando ésta es falsa, siendo cierta $H_{0}$, equivale a un error tipo I $(\alpha)$ o falso positivo, mientras que el rechazo de $H_{A}$ cuando ésta es cierta, siendo falsa $H_{0}$ equivale a un error tipo II $(\beta)$ o falso negativo $(1-\alpha$, corresponde a la sensibilidad de la prueba). A partir del error de tipo II se define el poder estadístico (potencia) del estudio, como la probabilidad $(1-\beta)$ de aceptar $\mathrm{H}_{\mathrm{A}}$ cuando es cierta (es- pecificidad de la prueba). También se define la potencia como la capacidad que posee una prueba estadística para detectar diferencias significativas de una cierta magnitud. La potencia dependerá en gran medida del tamaño muestral (Monleón, 2005; 2008).

La estadística se divide en dos partes principalmente:

1) La estadística descriptiva, que estudia los métodos de recogida, descripción, visualización y sintesis de los datos del estudio. Los datos pueden ser resumidos numérica o gráficamente. Algunos ejemplos de parámetros estadísticos serían la media y la desviación estándar, ejemplos gráficos son el histograma, diagramas de líneas, pirámide de población, clusters, etc.

2) La inferencia estadistica estudia la generación de modelos, inferencias y predicciones asociadas a los diferentes fenómenos estudiados. Se utiliza para realizar modelos 0 patrones en los datos y extraer inferencias sobre la población en estudio. Estas inferencias pueden ser respuestas a preguntas del tipo si/no (pruebas o test de hipótesis), estimaciones de características numéricas (estimación), pronósticos de futuras observaciones, descripciones de asociación numérica (correlación) o relaciones entre variables (análisis de regresión, análisis de la varianza (anova), series temporales, etc).

La estadistica inferencial trata de establecer conclusiones relacionadas con la población a través de extrapolar los resultados obtenidos de la muestra a la población de procedencia. Las caracteristicas que definen a la población se denominan parámetros poblacionales y las que definen a la muestra, estadísticos muestrales o estimadores (Ejemplo: medida de la probabilidad $(P)$ de incidencia de una enfermedad y su estimador la frecuencia relativa $\left(f_{r}\right)$ de la enfermedad).

Las ramas descriptiva e inferencial componen la estadística aplicada. Hay también una disciplina denominada estadística matemática, la cual representa sus bases teóricas (Wikipedia, 2009a).

Un modelo frecuente en muchos tipos de variables utilizadas habitualmente en los estudios indica que existe una tendencia de los valores alrededor de la media y menos observaciones a medida que nos acercamos a 
los extremos del rango de valores. Si el número $(n)$ de observaciones es grande, las distribuciones de frecuencia adoptan una forma de campana: campana de Gauss o distribución normal. Es una función continua que tiende asintóticamente a infinito por los extremos y cuyos valores $X$, se estandarizan a valores $Z$ en una función normal de media 0 y desviación estándar 1 , $\mathrm{N}(0,1)$ (Figura 1) .

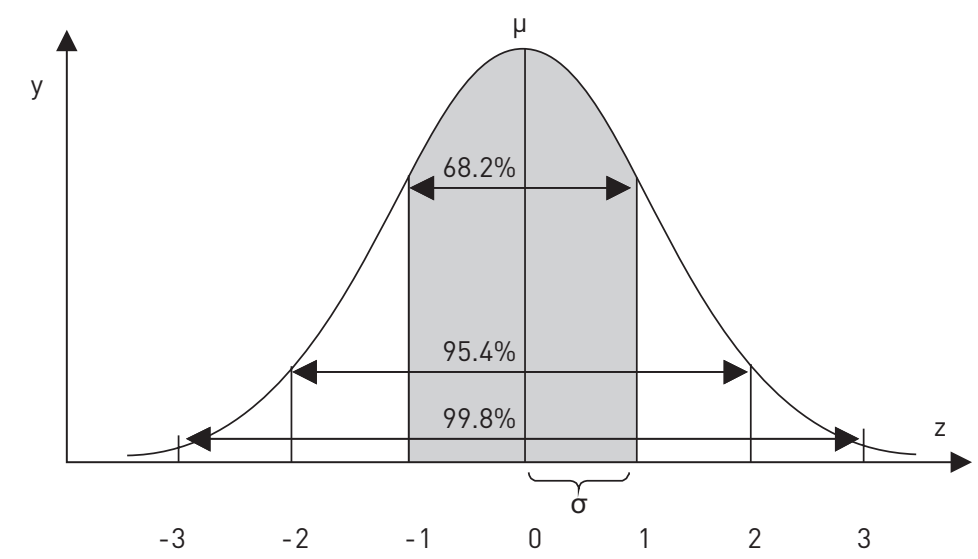

Figura 1. Valores de probabilidad de una función normal estandarizada $\mathrm{N}(0,1)$

A partir de la información aprovechable, el análisis estadístico pretende obtener una serie de conclusiones generales sobre la población, formulando una o varias hipótesis acerca de los datos. La estadística establece criterios de aceptación o no de las hipótesis que se plantean a partir de los datos, en términos de probabilidad.

Es necesario conocer el tipo de distribución de probabilidad de la población para poder estimar una probabilidad $(P)$ que ofrezca un criterio para aceptar o no la hipótesis nula.

El P-value puede entenderse como una medida de la disconformidad de los datos con la hipótesis nula. P-value suele ser utilizado ampliamente por los investigadores y se define la siguiente regla (En base a $\alpha=0,05$ ):

Si P-value $\leq 0,05 \rightarrow$ Se decide $\mathrm{H}_{\mathrm{A}}$

Si P-value $>0,05 \rightarrow$ Se decide $\mathrm{H}_{0}$

Cuando se desea determinar si un tratamiento es diferente de otro, se habla de prueba bilateral y por el contrario si se desea demostrar que un tratamiento es mejor que otro, se habla de prueba unilateral. El valor de P-value en un test de 2 colas es el doble del de un test de 1 cola.
Es ampliamente utilizado para representar significaciones la siguiente notación (Monleón, 2005):

* P-value $\leq 0,05$ (Resultados significativos)

** P-value $\leq 0,01$ (Resultados muy significativos)

*** P-value $\leq 0,001$ (Resultados altamente significativos).

Cuando comparamos la efectividad de dos tratamientos $\mathrm{A}$ y $B$ para tratar una determinada enfermedad puede que no sepamos cómo actúan farmacológicamente los tratamientos, pero si constatamos una asociación estadística más favorable con el tratamiento $A$ que con el $B$, y esto lo hacemos con una probabilidad de error inferior al $5 \%(\alpha)$, por lo que convenimos que la asociación no se debe sólo al azar (relación azarosa). Contrariamente si dado el caso de necesitar uno de los tratamientos y acudimos a un médico que desconoce las limitaciones del método estadístico y que sabe a ciencia cierta que el tratamiento $A$ es superior al $B$ para tratar nuestra enfermedad y cambia al paciente el tratamiento $B$ que estaba tomando por el $A$, podría suceder que al paciente le resultara más efectivo el tratamiento $B$ porque nos encontramos en ese 5\% erróneo, y sin embargo el médico se negaría a prescribirnos el tratamiento $B$ al considerar que ésto se debe a un efecto psicológico $u$ otra causa (Sánchez de Cos, 2007). 


\section{LOS ESTUDIOS UNIVERSITARIOS Y LOS PROFESIONALES DE LA ESTADÍSTICA}

Actualmente todas las carreras científicas, técnicas o humanistas se ofrecen asignaturas de estadística o relacionadas con ellas. Se suele introducir al alumno en el concepto de probabilidad y de variable aleatoria, explicando qué son las funciones de distribución y densidad de una variable aleatoria, se estudian típicamente la frecuencia, media, mediana, moda, varianza y desviación típica, población y muestra, comentando especialmente las propiedades de la distribución normal (gausiana) y el uso las diferentes pruebas estadísticas o técnicas como la regresión o el análisis de la varianza.

En el Libro Blanco del Grado de Estadística (ANECA, 2004) se hace una buena introducción a la historia de los estudios de estadística en Europa y España, así el comienzo de la Estadística en Europa se relaciona con el estudio de juegos de azar de mediados del siglo XVII, piénsese que en esa época el juego era considerado como una parte más de lo social, es decir en una forma más de expresión y comunicación humana. En 1654 se le propuso a Blaise Pascal (1623-1662) el problema siguiente "Una partida entre dos jugadores de igual habilidad se interrumpe por cualquier razón antes de llegar a su fin. A la vista de los tantos hasta el momento de la interrupción, y del tanteo total exigido para adjudicar el triunfo, ¿en qué proporción deberá dividirse el dinero en juego?" Pascal envió el problema a su amigo Pierre de Fermat (1601-1665) a través de la correspondencia que mantenían y entre ambos crearon para resolverlo, la Teoría de Probabilidades (Newman,1962).

La elaboración de estadísticas oficiales durante el siglo XIX y su secuela en el avance del bienestar logrado mediante la aplicación de políticas públicas eficientes fue un enorme impulso para ahondar de forma rigurosa de tipo de conocimiento. La Royal Statistical Society fue fundada en 1834 y en 1839 lo fue la American Statistical Association. El International Statistical Institute (ISI) fue fundado en 1885 y es hoy por hoy una de las asociaciones científicas más antiguas aún activas, su primer congreso tuvo lugar en 1853. ISI, como instituto autónomo, se ocupa de desarrollar y promover acciones de mejora de los métodos estadísticos y de su aplicación, así su éxito debe imputarse a "la creciente demanda mundial de información estadística profesional, a su sostenido liderazgo en el desarrollo de métodos y aplicaciones de la estadística y a la dedicación colectiva de sus miembros pertenecientes a 133 países" (ANECA, 2004). Es en estas fechas del siglo XIX cuando se inicia la difusión y estructuración de la enseñanza de las técnicas y métodos estadísticos en la universidad.

En el Reino Unido las primeras enseñanzas en Estadística Aplicada estuvieron vinculadas a la Demografía y la Salud Pública. Este horizonte explica el origen y la evolución de lo que actualmente nos encontramos como estudios universitarios europeos en Estadística (ANECA, 2004).

Actualmente en España, existen dos titulaciones oficiales de Estadistica: Diplomado en Estadística (titulación de primer ciclo, creada en 20 de diciembre de 1990, que se imparte en 13 universidades españolas de 7 comunidades autónomas) y Licenciado en Ciencias y Técnicas Estadísticas (título de segundo ciclo, de 6-8 de diciembre de 1994, que se imparte en 10 universidades de 7 comunidades autónomas) (ANECA, 2009).

En el curso 2009/2010 se inician en España los nuevos estudios del Grado de Estadística que según ANECA(2009) adaptados al Espacio Europeo de Enseñanaza Cómún (EEEC), denominado comúnmente Proceso de Bolonia. Asi el título de Grado en Estadística que se inicia en España en el curso 2009/2010 tiene como objetivo "la formación de profesionales capacitados para aplicar los métodos y modelos de la Estadística y la Investigación Operativa, asi como para realizar una gran cantidad de tareas específicas que acompañan a cualquier proceso de análisis de datos, que a menudo es un primer paso para preparar la toma de decisiones". El título de Grado en Estadística cubre una demanda social especifica de estadísticos profesionales y es homologable con títulos similares de los países más avanzados de nuestro entorno socioeconómico, especialmente Europa. Este título de Grado se orienta al mercado laboral en el que se observa una creciente demanda de titulados.

Cada vez con más frecuencia no solo la investigación científica universitaria sino la empresa privada se abre al campo de la estadística. Y es normal, si se tiene en cuenta que los grandes progresos empresariales acontecen por adelantarse a las acciones de su competencia, y la mejor estrategia es analizar el mercado y saber cómo va a responder ante un producto novedoso. 
La oferta de empleo público para estos profesionales es extensa, por ejemplo en algunos ministerios tienen un cuerpo específico de Técnicos Estadísticos, así como en las Administraciones autonómicas y las locales, también pueden ocupar puestos en diferentes áreas técnicas de la empresa, gracias a sus conocimientos matemáticos, y en áreas de marketing y comerciales, gracias a su conocimiento del mercado. Existen actualmente numerosas empresas de servicios para la industria farmacéutica que colaboran con los laboratorios farmacéuticos en el análisis de los datos de los desarrollos de nuevas moléculas farmacéuticas.

\section{Conclusiones}

Es imposible entender una sociedad moderna o Sociedad de la Información, sin la estadistica, ya que es la ciencia que ayuda a conocer y predecir el comportamiento de los individuos en su conjunto a partir de una muestra. Esto facilita conocer la intención de voto, las posibilidades de comercialización de un producto o si tendrá éxito un medicamento antes de que salga al mercado.

En esta situación se hace evidente la necesidad de disponer de profesionales encargados de la planificación, diseño, registro, selección, ordenación, síntesis y tratamiento específico de la información para que ésta se convierta en una herramienta útil que dé soporte a la toma de decisiones. Así la demanda actual de expertos en Estadística es muy alta y va en aumento, habiéndose convertido la demanda social de la Estadística en un indicador del nivel de desarrollo de los países (ANECA, 2004).

A pesar de que imposible entender una sociedad moderna sin estadística, es preocupante la situación actual de las ciencias matemáticas y más concretamente de la estadística en España, con un descenso año a año del número de estudiantes de estadística y matemáticas desde hace unos
10 años, aproximadamente, ya que a pesar de su imprescindibilidad y de las salidas profesionales en la Sociedad de la Información en la que vivimos, existe una falta de vocación entre los jóvenes por su estudio y uso, ya sea por su dificultad matemática u otros motivos. No creo que haya una crisis real de "vocaciones científicas", aunque sí han cambiado los términos del cálculo coste-beneficio entre el esfuerzo de estudiar una carrera técnica o científica y el sueldo obtenido, con lo que reivindico desde aquí la mejora de éstos profesionales imprescindibles, ya que en la actualidad se exporta más talento científico del que se importa. Estas impresiones vienen confirmadas por un reciente estudio "¿Hay una "crisis de vocaciones" científico-técnicas? El tránsito de la enseñanza secundaria a la universidad", presentado en el 2004 en Madrid el informe titulado cuya realización fue encargada por la Fundación Española para la Ciencia y la Tecnología (FECYT). El objetivo del informe era analizar la evolución de las preferencias hacia las diversas ramas de estudios de los alumnos que están acabando la enseñanza secundaria y comenzando la universitaria, en el período 1988-2001. Los resultados son interesantes para indicarnos las tendencias de las preferencias de la juventud española hacia unos u otros tipos de "saberes" (Zamora-Bonilla, 2004).

El Estadístico es por tanto un profesional que registra y analiza datos biológicos, médicos, económicos, sociológi$\cos$, físicos o de marketing a través de estudios, encuestas y observaciones. Con ellos, confecciona aclaraciones de lo que puede pasar en el futuro. Los datos de esas pesquisas, ordenados y depurados, se tornan estudios y gráficos donde se muestran los resultados y conclusiones que se derivan. De esta manera, la estadística y el análisis de los datos propician el desarrollo de las nuevas tecnologías de la información, exigiendo disponer de datos previamente registrados y generando nuevos datos, nuevo conocimiento, y por tanto información nueva y útil a la sociedad que permite su avance y la mejora de la calidad de nuestras vidas. 


\section{BIBLIOGRAFÍA}

ANECA (Agencia Nacional de Evaluación de la Calidad y Acreditación) (2009): Título de grado en estadística (disponible en: http://www.aneca.es/media/150324/ libroblanco_estadistica_def.pdf).

Bernabeu-Mestre, J. (2007): "Estadística y salud pública: el argumento del método numérico", Gac Sanit (21) 5

Mahalanobis, P. C. (1954): "The foundations of Statistics", Dialectica, 8, 95-11.

Monleón, T., Barnadas, A., Roset, M. (2005): Metodología de investigación y estadística en oncología y hematología, Módulo 5, p. 6-26. ISBN: B-40.747.2005. Ed Edimac Barcelona.

Monleón-Getino, Toni; Barnadas-Molins, Agustí y Roset-Gamisans, Montserrat: "Diseños secuenciales y análisis intermedio en la investigación clínica: tamaño versus dificultad", Medicina Clínica, 2008; 132 (11): 437-442.

Newman, J. R. (1962): ¿Qué es la ciencia?. Difución científica, Aguilar, Madrid, pp. 52-53.

Recibido: 10 de septiembre de 2009 Aceptado: 1 de febrero de 2010
Rubio de Juan, M. A. (2002): Lección Inaugural: Estadistica, Certeza e Incertidumbre, Lección inaugural del curso académico 2001/2002 de la Universidad de Extremadura.

Salinas, P. J.: La estadística: disforia en ciencias de la salud, MedULA (16) 2.

Sanchez de Cos (2007): La trastienda de la estadística. De todo un poco, blog electrónico, disponible en: http:// ernestosdc.wordpress.com/category/ estadistica/.

Tanur, J. M., Mosteller, F., et al. Eds. (1992). La Estadística: Una guía de lo desconocido. Alianza. Madrid.

Wikipedia (2009a): La estadistica, disponible en: http://es.wikipedia.org/wiki/ Estad\%C3\%ADstica.

Wikipedia (2009b): Aleatoriedad, disponible en: http://es.wikipedia.org/wiki/ Aleatoriedad.

Zamora-Bonilla (2004): ¿Hay una "crisis de vocaciones" científico-técnicas? El tránsito de la enseñanza secundaria a la universidad, FECYT (disponible on-line en: http://abordodelottoneurath.blogspot.com/2009/06/ la-crisis-de-vocaciones-cientificasun.html. 